\title{
Excited States and Their Dynamics in CdSe Quantum Dots Studied by Two-Color 2D Spectroscopy
}

Wang, Zhengjun; Lenngren, Nils; Amarotti, Edoardo; Hedse, Albin; Žídek, Karel; Zheng, Kaibo; Zigmantas, Donatas; Pullerits, Tônu

Published in:

Journal of Physical Chemistry Letters

Link to article, DOI:

10.1021/acs.jpclett.1c04110

Publication date:

2022

Document Version

Publisher's PDF, also known as Version of record

Link back to DTU Orbit

Citation $(A P A)$ :

Wang, Z., Lenngren, N., Amarotti, E., Hedse, A., Žídek, K., Zheng, K., Zigmantas, D., \& Pullerits, T. (2022).

Excited States and Their Dynamics in CdSe Quantum Dots Studied by Two-Color 2D Spectroscopy. Journal of Physical Chemistry Letters, 13(5), 1266-1271. https://doi.org/10.1021/acs.jpclett.1c04110

\section{General rights}

Copyright and moral rights for the publications made accessible in the public portal are retained by the authors and/or other copyright owners and it is a condition of accessing publications that users recognise and abide by the legal requirements associated with these rights.

- Users may download and print one copy of any publication from the public portal for the purpose of private study or research.

- You may not further distribute the material or use it for any profit-making activity or commercial gain

- You may freely distribute the URL identifying the publication in the public portal 


\title{
Excited States and Their Dynamics in CdSe Quantum Dots Studied by Two-Color 2D Spectroscopy
}

\author{
Zhengjun Wang, Nils Lenngren, Edoardo Amarotti, Albin Hedse, Karel Žídek, Kaibo Zheng, \\ Donatas Zigmantas, and Tõnu Pullerits*
}

Cite This: J. Phys. Chem. Lett. 2022, 13, 1266-1271

Read Online

\begin{abstract}
ACCESS |
山ll Metrics \& More

回 Article Recommendations

ABSTRACT: Quantum dots (QDs) form a promising family of nanomaterials for various applications in optoelectronics. Understanding the details of the excited-state dynamics in QDs is vital for optimizing their function. We apply two-color 2D electronic spectroscopy to investigate $\mathrm{CdSe} \mathrm{QDs}$ at $77 \mathrm{~K}$ within a broad spectral range. Analysis of the electronic dynamics during the population time allows us to identify the details of the excitation pathways. The initially excited high-energy electrons relax with the time constant of $100 \mathrm{fs}$. Simultaneously, the states at the band edge rise within 700 fs. Remarkably, the excitedstate absorption is rising with a very similar time constant of $700 \mathrm{fs}$. This makes us reconsider the earlier interpretation of the excited-state absorption as the signature of a long-lived trap state. Instead, we propose that this signal originates from the excitation of the electrons that have arrived in the conduction-band edge.
\end{abstract}

S1 Supporting Information

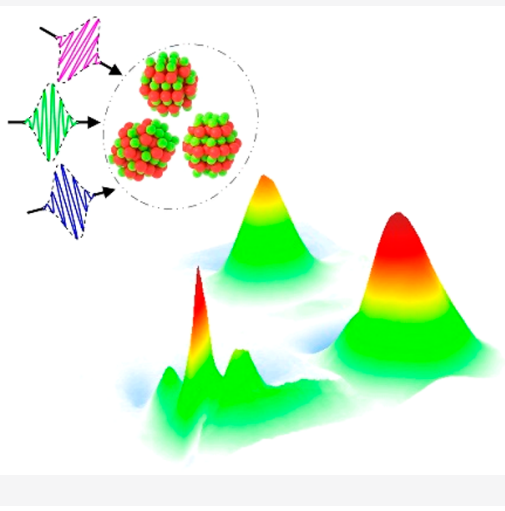

$\mathrm{T}$ he discovery of the quantum size effect in colloidal semiconductor nanocrystals, ${ }^{1,2}$ the so-called quantum dots (QDs), opened a new topic in nanomaterial research. An important milestone of the following development was the introduction of the hot-injection method, enabling easy synthesis of high-quality monodisperse QDs. ${ }^{3}$ Since then, the field has been expanding toward a broad combination of materials, sophisticated structures, and optoelectronic devices, ${ }^{4}$ such as light-emitting diodes ${ }^{5}$ and microspectrometers. ${ }^{6}$ Throughout the years, numerous studies have addressed a broad set of fundamental questions regarding excited states and their dynamics in QDs. ${ }^{7-12}$ Several recent studies have addressed issues like high-intensity effects ${ }^{13-16}$ and the influence of charging on excited-state dynamics ${ }^{17,14}$-all important from the point of view of possible optoelectronic applications of QDs.

Recent developments in coherent multidimensional spectroscopy (CMDS) ${ }^{18-27}$ have opened new possibilities for investigating excited-state dynamics with a very high level of precision in both time and spectral resolution. ${ }^{28,29}$ The method has demonstrated its capabilities in studies of dynamics, including coherent evolution of both vibrational and electronic origin in both biomaterials and semiconductors. ${ }^{30,31}$ It has become increasingly popular to investigate quantum coherence, relaxation, and coupling of excited states by using CMDS. ${ }^{28,30,32,33}$ Numerous studies on excited-state dynamics in QDs have applied coherent 2D spectroscopy. ${ }^{18,23,28,33,34}$

In this Letter, we extend our previous $2 \mathrm{D}$ spectroscopy study of CdSe QDs at $77 \mathrm{~K}^{23}$ by significantly lengthening the spectral coverage (now ranging from 15200 to $21300 \mathrm{~cm}^{-1}$ ) via applying additional pulse energies and thereby using twocolor $2 \mathrm{D}$ spectroscopy.

The peaks of the $2 \mathrm{D}$ spectra originate from many different optical responses, such as ground-state bleach (GSB), stimulated emission ( $S E$ ), and excited-state absorption (ESA). ${ }^{35,36}$ Furthermore, depending on the pulse ordering, we can distinguish the rephasing and nonrephasing signals, which provide complementary information. Using the breadth of available information, we obtain a detailed description of an extensive range of the CdSe QD states and their excitation dynamics during the first 1400 fs after excitation.

$2 \mathrm{D}$ electronic spectroscopy ${ }^{37}$ (2DES) is a third-order nonlinear optical method which uses three short laser pulses acting on the sample. Between these three pulses, there are two delay times in the 2DES experiment. However, since the coherently generated signal also provides an instance of fieldmatter interaction, in total we have three time intervals to count, namely coherence time $t_{1}$ (often noted as $\tau$, between the first and the second pulses), population time $t_{2}$ (often noted $T$, between the second and third pulses), and detection time $t_{3}$ (also called $t$, between the third pulse and the signal generation).

Received: December 18, 2021

Accepted: January 21, 2022

Published: January 28, 2022 


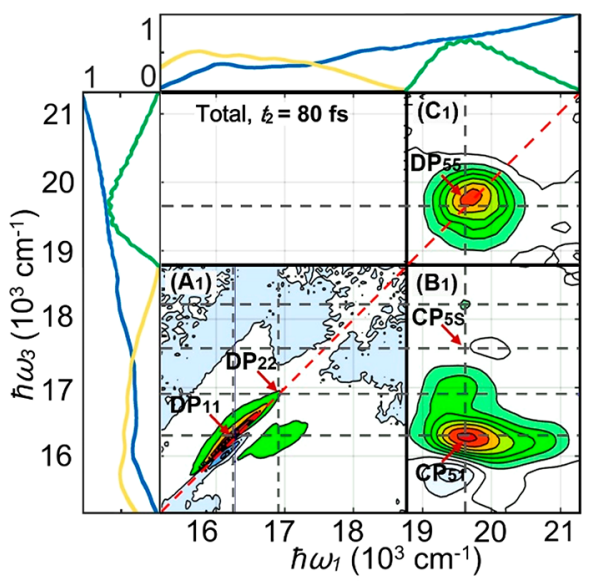

(a)

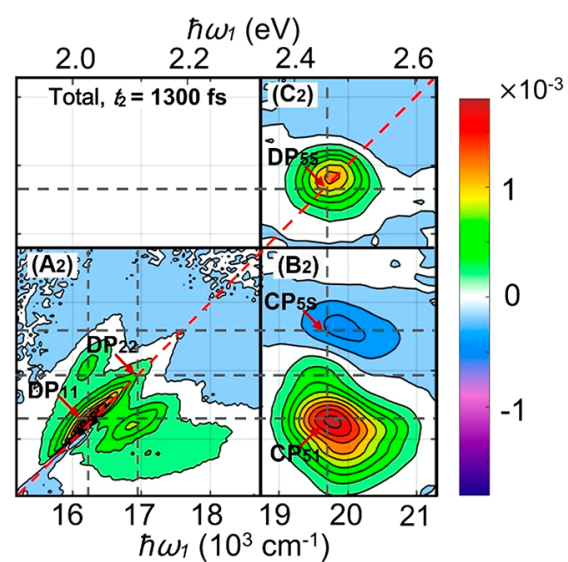

(b)

Figure 1. Real part of the total 2D spectroscopy signal at two population times, assembled from three separate single-color (A and C) and twocolor (B) measurements. The black horizontal dashed lines mark the positions of the states $\left|e_{1}\right\rangle,\left|e_{2}\right\rangle,\left|e_{3}\right\rangle,\left|e_{4}\right\rangle$, and $\left|e_{5}\right\rangle$ at $16200,16900,17800$, 18300 , and $19700 \mathrm{~cm}^{-1}$, respectively. The three vertical lines mark the states which lead to the clear cross peaks. The labeled diagonal peaks (DP) and cross peaks (CP) are discussed further in the text. The leftmost and topmost panels show the laser spectra (yellow and green curves) and the absorbance of CdSe QDs at $77 \mathrm{~K}$ (blue curves).

\section{Table 1. Excited States of CdSe QDs in the 2D Electronic Spectrum}

\begin{tabular}{llllll} 
excitonic states & \multicolumn{1}{c}{$\left|e_{1}\right\rangle^{23}$} & \multicolumn{1}{c}{$\left|e_{2}\right\rangle^{23}$} & \multicolumn{1}{c}{$\left|e_{3}\right\rangle^{23}$} & \multicolumn{1}{c}{$\left|e_{4}\right\rangle^{23}$} & $\left|e_{5}\right\rangle$ \\
symbols & $1 S_{3 / 2}(h)-1 S(e)$ & $2 S_{3 / 2}(h)-1 S(e)$ & $1 S_{1 / 2}(h)-1 S(e)$ & $1 P_{3 / 2}(h)-1 P(e)$ & $2 S_{1 / 2}(h)-1 S(e)$ \\
energy $\left(\mathrm{cm}^{-1}\right)$ & 16200 & 16900 & 17800 & 18300 & 19700 \\
\hline
\end{tabular}

In traditional 2D spectroscopy, the three pulses have the same carrier frequency, and consequently, the measurable energy region is the same in both spectral dimensions of the $2 \mathrm{D}$ map. In this work, we investigate the dynamics of the excited states of CdSe QDs by using pulses of different frequency, thereby broadening the spectral coverage of the experiment. This spectroscopic technique is called two-color 2D spectroscopy. ${ }^{38,39}$ In our two-color 2D spectroscopy measurement, the first two laser pulses (also called pump pulses) resonate with a high-energy state of the sample system, and the third laser pulse (also called the probe pulse) together with a local oscillator ( $\mathrm{LO}$ ) covers low-energy region near the band edge of the sample (for detailed experimental information, see the Supporting Information). That is the reason the $\omega_{1}$-axis and the $\omega_{3}$-axis are different in the two-color $2 \mathrm{D}$ spectrum shown in panels $\mathrm{B}_{1}$ and $\mathrm{B}_{2}$ of Figure 1 . The spectrogram reveals a rich network of cross peaks in the twocolor 2D spectrum. Two-color 2D spectroscopy shows more nondegenerate or off-diagonal traits, which provides knowledge on energy or coherence transitions between electronic levels. ${ }^{38,39}$ These peak networks reflect correlations between the excited states, and their population time dependence provides information about the dynamics among excited states in the CdSe QD system.

CdSe QDs were prepared as in our previous work; ${ }^{23}$ for details see the Supporting Information.

The measured 2D signals can be divided into low- and highenergy regions. The wavenumber ranges of the low- and highenergy regions are from 15200 to $18800 \mathrm{~cm}^{-1}$ and from 18800 to $21300 \mathrm{~cm}^{-1}$, respectively. In the experimental results as shown in Figure 1, the $x$-axis refers to the excitation energy $\left(\hbar \omega_{1}\right)$ and the $y$-axis corresponds the detection energy $\left(\hbar \omega_{3}\right)$. The $B_{1}$ and $B_{2}$ panels are the two-color parts (the wavenumber range of the excitation energy is from 18800 to $21300 \mathrm{~cm}^{-1}$; the detection energy range is from 15200 to $18800 \mathrm{~cm}^{-1}$ ). The
$\mathrm{A}_{2}, \mathrm{~B}_{2}$, and $\mathrm{C}_{2}$ panels correspond to the population time $t_{2}=$ $1300 \mathrm{fs}$. The red arrows point to the spectral features which are analyzed and discussed in detail. The labels DP and CP stand for diagonal peaks and cross peaks, respectively. To the left of and above the 2D plots, we show the absorption spectrum of the QDs (blue) and the pulse spectra (yellow and green).

We will discuss the three main diagonal peaks $\mathrm{DP}_{11}, \mathrm{DP}_{22}$, and $\mathrm{DP}_{55}$ and three of the cross peaks $\mathrm{CP}_{52}, \mathrm{CP}_{51}$, and $\mathrm{CP}_{5 \mathrm{~S}}$. The subscript $S$ represents the ESA signal possibly originating from a trap state as earlier discussed in ref 23, or it can be due to some other excited states. The two-color (off-diagonal) region contains the $\mathrm{CP}_{52}, \mathrm{CP}_{51}$, and $\mathrm{CP}_{5 \mathrm{~S}}$ peaks. Among the above peaks, the $\mathrm{DP}_{11}$ and $\mathrm{DP}_{22}$ peaks were part of the analysis by Lenngren et al. ${ }^{23}$ showing hole trapping from the corresponding states.

Here, we mainly analyze the relaxation dynamics in the highenergy region. We use the total and rephasing parts of the $2 \mathrm{D}$ spectrum to illustrate the details of excited-state transitions of CdSe QDs.

The excited-state dynamics of CdSe QDs are analyzed based on the total and rephasing $2 \mathrm{D}$ maps measured at $77 \mathrm{~K}$. The main excitonic states were identified based on Norris and Bawendi's work, ${ }^{40}$ extending the analysis in Lenngren et al. ${ }^{23}$ The measured excitonic states are shown in Table 1. For more details of these fits, refer to the Supporting Information.

We make use of the standard state nomenclature widely used for describing the excited states of QDs. ${ }^{40}$ The states are represented by a combination of the electron and hole principal quantum numbers and angular momentum states such as $1 S, 1 P$, and $1 D$, together with the total angular momentum term for holes, which is $3 / 2$ or $1 / 2$ for the states discussed here. ${ }^{41,40}$ There are five excited states of CdSe QDs identified in the spectral coverage of our 2D spectroscopy experiment. 
Let us take a closer look at the $\left|e_{5}\right\rangle$ state which originates from the $2 S_{1 / 2}(h)$ hole and the $1 S(e)$ electron. ${ }^{40}$ The $\mathrm{DP}_{55}$ peak mainly originates from this state (see the spectral fit in the Supporting Information) with negligible contribution from higher energy transitions like $3 S_{1 / 2}(h)-1 S(e){ }^{41,40}$ Figure 2

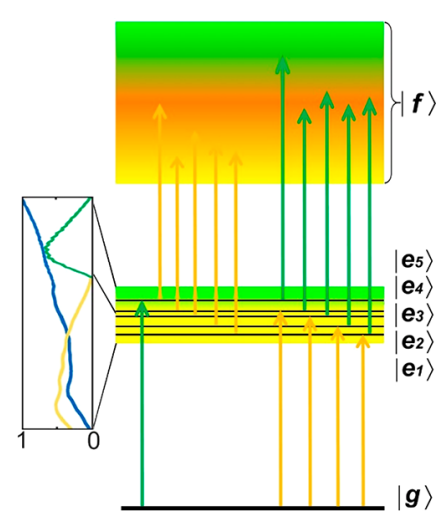

Figure 2. Singly and doubly excited energy levels together with the possible transitions that are driven by the pulses of two different energies. The yellow region is the low-energy area, and the green region is the high-energy area. In the left panel, the laser spectra are represented by yellow and green curves and the absorption of CdSe QDs as a blue curve. The yellow arrows indicate the transition processes due to the low-energy pulses. Similarly, the green arrows represent the possible transitions due to the high-energy pulses.

illustrates the possible transitions due to the above five excited states. To account for the ESA signal, we also need to consider the doubly excited manifold $|f\rangle$. The $|f\rangle$ manifold covers a wide range of transitions from the states $\left|e_{1}\right\rangle,\left|e_{2}\right\rangle,\left|e_{3}\right\rangle,\left|e_{4}\right\rangle$, and $\left|e_{5}\right\rangle$ and the possible other excited states like traps. The specific details are discussed in the following section and also shown in Figure 4.

In Figure 3, the peak $\mathrm{DP}_{11}$ mainly comes from the excited state $\left|e_{1}\right\rangle$ at around $16200 \mathrm{~cm}^{-1}$. Elongation of the peak $\mathrm{DP}_{11}$ along the diagonal of the $2 \mathrm{D}$ map originates from inhomogeneous broadening due to the size distribution of CdSe QDs. To further understand the relaxation dynamics of CdSe QDs among $\left|e_{1}\right\rangle,\left|e_{5}\right\rangle$, and other excited states, we analyze the evolution of the $2 \mathrm{D}$ spectra during the population time from 0 to $1400 \mathrm{fs}$. The $\mathrm{CP}_{51}, \mathrm{CP}_{52}$, and $\mathrm{CP}_{5 \mathrm{~s}}$ peaks, appearing in the lower right corner, and the $\mathrm{DP}_{55}$ peak, appearing in the upper right corner, are all related to the excitation of the state $\left|e_{5}\right\rangle$ at $19700 \mathrm{~cm}^{-1}$. ${ }^{40}$ The cross peaks $\mathrm{CP}_{51}$ and $\mathrm{CP}_{52}$ are visible already at early times (Figures $1 \mathrm{a}$ and $3 \mathrm{a}$ ), showing the correlation of states $\left|e_{5}\right\rangle,\left|e_{2}\right\rangle$, and $\left|e_{1}\right\rangle$. The dynamics seen in the population time dependence of $\mathrm{CP}_{51}, \mathrm{CP}_{5 \mathrm{~S}}$, and $\mathrm{DP}_{55}$ peaks in Figure 3 reflect the population relaxation through the ladder of the levels and represent the overall population dynamic from 21300 to $15500 \mathrm{~cm}^{-1}$. The structure at $\hbar \omega_{1}>20500 \mathrm{~cm}^{-1}$ might be due to signals from higher-energy states or the dispersive line shape of the $2 \mathrm{D}$ spectra associated with $\left|e_{5}\right\rangle$.

We follow the peak changes until $t_{2}=1400 \mathrm{fs}$. After that, no further changes occur apart from the overall decay of the excited state. ${ }^{23}$ To avoid the possible nonresonant signal components during the pulse overlap (see Figure S6), we start the analyses from $80 \mathrm{fs}$. The population time dependence of the most significant features of the real part of the $2 \mathrm{D}$ spectra is shown in Figure 4. The cuts of the three $2 \mathrm{D}$ peaks taken at $\left|e_{5}\right\rangle$ excitation are shown in Figure 4a. The cross peak $\mathrm{CP}_{51}$ corresponds to the detection of $\left|e_{1}\right\rangle$ and $\mathrm{DP}_{55}$ to the detection of $\left|e_{5}\right\rangle$, while the origin of the ESA component $\mathrm{CP}_{5 \mathrm{~s}}$ cannot be uniquely identified due to the uncertainty of the energy of the state responsible for ESA signals. The three kinetic traces in Figure $4 \mathrm{~b}$ show the population time dependence of the peaks $\mathrm{DP}_{55}, \mathrm{CP}_{5 \mathrm{~S}}$, and $\mathrm{CP}_{51}$, respectively. Each curve was fitted by an exponential function as shown in Figure $4 \mathrm{~b}$. The lifetimes obtained for these peaks are $100 \pm 10,700 \pm 50$, and $700 \pm 50$ fs, respectively. For more details, refer to the Supporting Information.

The decaying diagonal peak $\mathrm{DP}_{55}$ is well described by the GSB and SE pathways (see Figure 4c). The contribution of SE gradually decreases in $\mathrm{DP}_{55}$ since the population is relaxing. The $100 \mathrm{fs}$ decay, therefore provides the relaxation time of the initially created $\left|e_{5}\right\rangle$. The contribution of GSB to $\mathrm{DP}_{55}$ is nearly constant within the measured population time; see the corresponding Feynman diagrams. The signal lives as long as the ground state is recovered. The growth of the band $\mathrm{CP}_{5 S}$ corresponds to the arrival of the initial $\left|e_{5}\right\rangle$ population in a state which absorbs at about $17500 \mathrm{~cm}^{-1}$. The subscript $S$ indicates that we cannot uniquely identify the state based on the ESA signal only. For example, in our earlier work, ${ }^{23}$ the analogous signal at lower energy excitation was assigned to a trap state. The band $\mathrm{CP}_{51}$ is mainly due to the GSB(iii) and $\mathrm{SE}(\mathrm{iv})$

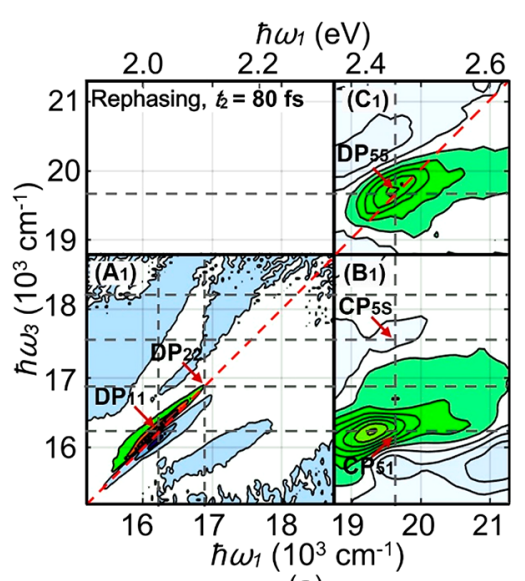

(a)

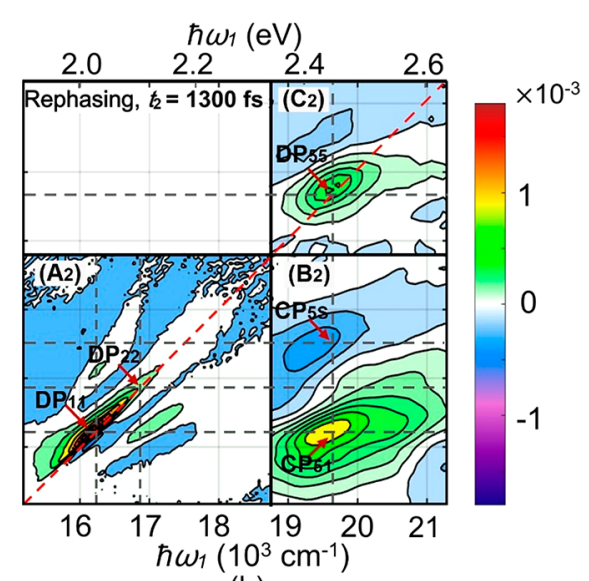

(b)

Figure 3. Real part of the rephasing $2 \mathrm{D}$ spectrum at two representative time points, assembled from two single-color (A, C) and a two-color (B) measurement. 

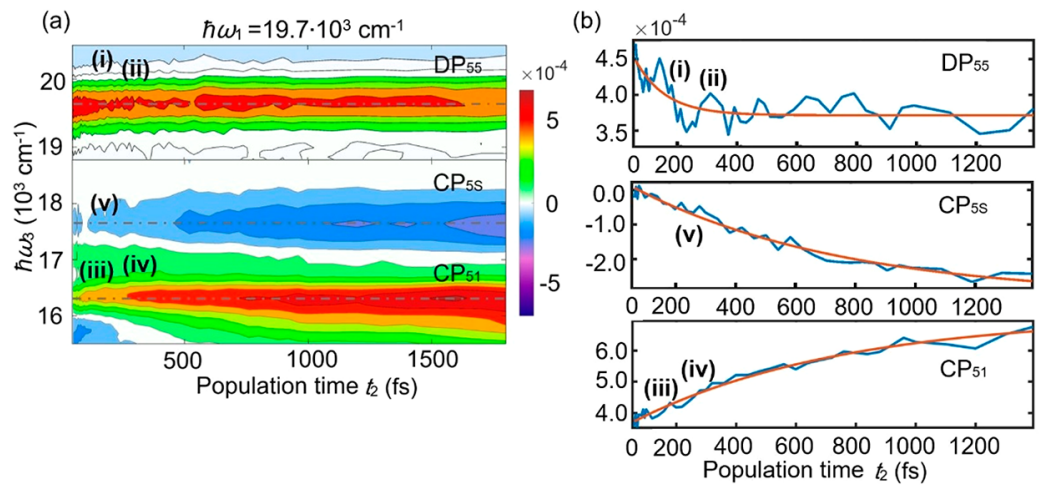

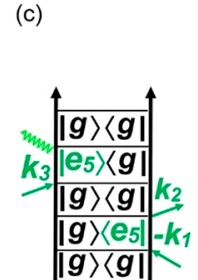

(i) $\mathrm{DP}_{55}: \mathrm{GSB}$

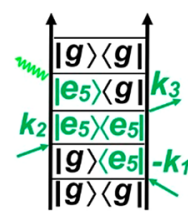

(ii) $\mathrm{DP}_{55}: \mathrm{SE}$

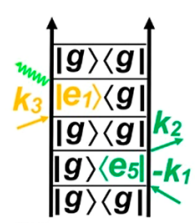

(iii) $\mathrm{CP}_{51}$ :GSB

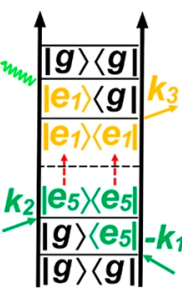

(iv) $\mathrm{CP}_{51}: \mathrm{SE}$

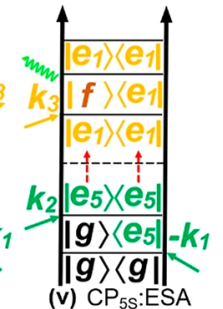

Figure 4. Population-time dependence of the main spectral features. (a) The real part of the three main peaks of the rephasing 2D spectra as a function of the population time $t_{2}$. (The results without normalization can be found in the Supporting Information.) (b) Kinetics of the three main peaks $\mathrm{DP}_{55}, \mathrm{CP}_{5 S}$, and $\mathrm{CP}_{51}$. The exponential decay and rise times of the three curves are $100 \pm 10,700 \pm 50$, and $700 \pm 50 \mathrm{fs}$, respectively. (c). Feynman diagrams (i) and (ii) are the GSB and SE pathways for the $\mathrm{DP}_{55}$ peak. Feynman diagram (iii) represents the GSB pathway for the $\mathrm{CP}_{51}$ peak. Feynman diagram (v) indicates the relaxation processes of $\mathrm{CP}_{5 \mathrm{~S}}$.

pathways. While the GSB is constant, the SE grows with time. The corresponding time constant is $700 \mathrm{fs}$, telling us the time it takes for the $\left|e_{5}\right\rangle$ excitation to relax to $\left|e_{1}\right\rangle$. Because this is very similar to the growth of the $\mathrm{CP}_{5 \mathrm{~S}}$, we conclude that both bands originate from the same state, also suggesting a reinterpretation of the ESA feature around $\hbar \omega_{1}=18300 \mathrm{~cm}^{-1}$ and $\hbar \omega_{3}=$ $17500 \mathrm{~cm}^{-1}$ (labeled $\mathrm{F}$ in our previous work ${ }^{23}$ ).

At first glance, the time constants seem to contradict-we say that the relaxation from $\left|e_{5}\right\rangle$ takes $100 \mathrm{fs}$, but the arrival to $\left|e_{1}\right\rangle$ corresponds to a $700 \mathrm{fs}$ time constant. To explain this, we need to consider the relaxation mechanism-coupling to the LO phonons with the frequency of about $200 \mathrm{~cm}^{-1}$ in CdSe. Because the electronic relaxation gives energy away to the nuclear lattice, this phonon quantum is the largest possible relaxation step. The higher-order multiphonon steps are significantly less likely to occur. The energy gap between $\left|e_{5}\right\rangle$ and $\left|e_{1}\right\rangle$ is $3400 \mathrm{~cm}^{-1}$. Thus, it takes over 15 jumps to relax from the $\left|e_{5}\right\rangle$ to the $\left|e_{1}\right\rangle$ state. This brings up another contradiction-the energy gap between the QD states is far larger than the phonon energy, suggesting a drastic slowdown of the relaxation. The expected reduction of the relaxation in QDs is called the phonon bottleneck. As explained by atomistic calculations $^{42}$ of the QD electronic band structure, even though the spectral features follow the effective-mass theory nomenclature that we use, in reality, the QD electronic bands are quasi-continuous with a significant number of states to provide efficient phonon-induced relaxation pathways down to the band edge. When exciting into $\left|e_{5}\right\rangle$, we do not rule out hole trapping from $\left|e_{1}\right\rangle$ as described by our previous work ${ }^{23}$ as part of a multistep relaxation pathway, but it is not observable directly in the two-color data due to the strong $\mathrm{CP}_{51}$ peak.

The two-color $2 \mathrm{D}$ spectroscopy provides additional spectral coverage and thereby allows access to the relaxation dynamics from higher excited states in QDs. A diagonal peak $\left(\mathrm{DP}_{55}\right)$ and three cross peaks $\left(\mathrm{DP}_{11}, \mathrm{CP}_{51}\right.$, and $\left.\mathrm{CP}_{5 \mathrm{~S}}\right)$ were observed by
2DES in this region. We clarified the relaxation dynamics in QDs based on these main 2D spectral bands. The relaxation occurs through the coupling to the LO phonons, and it takes multiple consecutive relaxation jumps to reach the low-energy band edge. The relaxation process takes about $700 \mathrm{fs}$.

\section{ASSOCIATED CONTENT}

\section{SI Supporting Information}

The Supporting Information is available free of charge at https://pubs.acs.org/doi/10.1021/acs.jpclett.1c04110.

Additional material explaining details of the sample preparation, 2DES setup and the experimental conditions, further experimental results, energy level system of CdSe QDs, and data processing (PDF)

\section{AUTHOR INFORMATION}

\section{Corresponding Author}

Tõnu Pullerits - Division of Chemical Physics and NanoLund, Lund University, 22100 Lund, Sweden; (1) orcid.org/0000-0003-1428-5564; Email: tonu.pullerits@chemphys.lu.se

\section{Authors}

Zhengjun Wang - Division of Chemical Physics and NanoLund, Lund University, 22100 Lund, Sweden; (1) orcid.org/0000-0002-7599-0382

Nils Lenngren - Division of Chemical Physics and NanoLund, Lund University, 22100 Lund, Sweden; ELI Beamlines, Institute of Physics, Czech Academy of Sciences, 25241 Dolní Břžany, Czech Republic

Edoardo Amarotti - Division of Chemical Physics and NanoLund, Lund University, 22100 Lund, Sweden

Albin Hedse - Division of Chemical Physics and NanoLund, Lund University, 22100 Lund, Sweden 
Karel Žídek - Division of Chemical Physics and NanoLund, Lund University, 22100 Lund, Sweden; Regional Center for Special Optics and Optoelectronic Systems (TOPTEC), Institute of Plasma Physics of the Czech Academy of Sciences, 27000 Prague 8, Czech Republic

Kaibo Zheng - Division of Chemical Physics and NanoLund, Lund University, 22100 Lund, Sweden; Department of Chemistry, Technical University of Denmark, DK-2800 Kongens Lyngby, Denmark; 이이이.org/0000-0002-72361070

Donatas Zigmantas - Division of Chemical Physics and NanoLund, Lund University, 22100 Lund, Sweden; (1) orcid.org/0000-0003-2007-5256

Complete contact information is available at: https://pubs.acs.org/10.1021/acs.jpclett.1c04110

\section{Author Contributions}

Z.W. and N.L. contributed equally to this work. All authors have read and agree with the final manuscript.

\section{Notes}

The authors declare no competing financial interest.

\section{ACKNOWLEDGMENTS}

This work was financed by the Swedish Research Council (VR), China Scholarship Council, NanoLund, the Knut and Alice Wallenberg Foundation (Project 2016.0089), an Independent Research Fund Denmark-Sapere Aude starting grant (no. 7026-00037A), the Crafoord Foundation (no. 20200522), Research Fund for International Young Scientists from NSFC, China (no. 21950410515), the Swedish Energy Agency, the ADONIS project (CZ.02.1.01/0.0/0.0/16_019/ 0000789) of the European Regional Development Fund and the Ministry of Education, Youth and Sports of the Czech Republic, and the European Union's Horizon 2020 research and innovation program under the Marie Skłodowska-Curie grant agreement no. 945378. We thank Egle Bukarte and Pavel Kolesnichenko for discussions and handling of the experimental data. We also thank Mohamed A. Abdellah for help in synthesizing samples.

\section{REFERENCES}

(1) Rossetti, R.; Nakahara, S.; Brus, L. E. Quantum Size Effects in the Redox Potentials, Resonance Raman Spectra, and Electronic Spectra of CdS Crystallites in Aqueous Solution. J. Chem. Phys. 1983, 79 (2), 1086-1088.

(2) Ekimov, A. I.; Efros, A. L.; Onushchenko, A. A. Quantum Size Effect in Semiconductor Microcrystals. Solid State Commun. 1993, 88, 947-950.

(3) Murray, C. B.; Norris, D. J.; Bawendi, M. G. Synthesis and Characterization of Nearly Monodisperse CdE $(E=S$, Se, Te) Semiconductor Nanocrystallites. J. Am. Chem. Soc. 1993, 115 (19), 8706-8715.

(4) Meinardi, F.; Colombo, A.; Velizhanin, K. A.; Simonutti, R.; Lorenzon, M.; Beverina, L.; Viswanatha, R.; Klimov, V. I.; Brovelli, S. Large-Area Luminescent Solar Concentrators Based on Stokes-ShiftEngineered Nanocrystals in a Mass-Polymerized PMMA Matrix. Nat. Photonics. 2014, 8 (5), 392-399.

(5) Kim, T.; Kim, K. H.; Kim, S.; Choi, S. M.; Jang, H.; Seo, H. K.; Lee, H.; Chung, D. Y.; Jang, E. Efficient and Stable Blue Quantum Dot Light-Emitting Diode. Nature. 2020, 586 (7829), 385-389.

(6) Bao, J.; Bawendi, M. G. A Colloidal Quantum Dot Spectrometer. Nature. 2015, 523 (7558), 67-70.

(7) Klimov, V. I. Mechanisms for Photogeneration and Recombination of Multiexcitons in Semiconductor Nanocrystals: Implications for Lasing and Solar Energy Conversion. J. Phys. Chem. B 2006, 110 (34), 16827-16845.

(8) Klimov, V. I.; Ivanov, S. A.; Nanda, J.; Achermann, M.; Bezel, I.; McGuire, J. A.; Piryatinski, A. Single-Exciton Optical Gain in Semiconductor Nanocrystals. Nature. 2007, 447 (7143), 441-446.

(9) Schaller, R. D.; Agranovich, V. M.; Klimov, V. I. High-Efficiency Carrier Multiplication through Direct Photogeneration of MultiExcitons via Virtual Single-Exciton States. Nat. Phys. 2005, 1 (3), 189-194.

(10) Kambhampati, P. Unraveling the Structure and Dynamics of Excitons in Semiconductor Quantum Dots. Acc. Chem. Res. 2011, 44 (1), 1-13.

(11) Huang, J.; Huang, Z.; Yang, Y.; Zhu, H.; Lian, T. Multiple Exciton Dissociation in CdSe Quantum Dots by Ultrafast Electron Transfer to Adsorbed Methylene Blue. J. Am. Chem. Soc. 2010, 132 (13), 4858-4864.

(12) Kambhampati, P. Hot Exciton Relaxation Dynamics in Semiconductor Quantum Dots: Radiationless Transitions on the Nanoscale. J. Phys. Chem. C 2011, 115 (45), 22089-22109.

(13) Žídek, K.; Zheng, K.; Abdellah, M.; Lenngren, N.; Chábera, P.; Pullerits, T. Ultrafast Dynamics of Multiple Exciton Harvesting in the $\mathrm{CdSe}-\mathrm{ZnO}$ System: Electron Injection versus Auger Recombination. Nano Lett. 2012, 12 (12), 6393-6399.

(14) Honarfar, A.; Chabera, P.; Lin, W.; Meng, J.; Mourad, H.; Pankratova, G.; Gorton, L.; Zheng, K.; Pullerits, T. Ultrafast Spectroelectrochemistry Reveals Photoinduced Carrier Dynamics in Positively Charged CdSe Nanocrystals. J. Phys. Chem. C 2021, 125 (26), 14332-14337.

(15) Shulenberger, K. E.; Coppieters 't Wallant, S. C.; Klein, M. D.; McIsaac, A. R.; Goldzak, T.; Berkinsky, D. B.; Utzat, H.; Barotov, U.; Van Voorhis, T.; Bawendi, M. G. Resolving the Triexciton Recombination Pathway in CdSe/CdS Nanocrystals through StateSpecific Correlation Measurements. Nano Lett. 2021, 21 (18), 74577464.

(16) Kroupa, D. M.; Pach, G. F.; Vörös, M.; Giberti, F.; Chernomordik, B. D.; Crisp, R. W.; Nozik, A. J.; Johnson, J. C.; Singh, R.; Klimov, V. I.; Galli, G.; Beard, M. C. Enhanced Multiple Exciton Generation in PbSICdS Janus-like Heterostructured Nanocrystals. ACS Nano 2018, 12, 10084-10094.

(17) Honarfar, A.; Mourad, H.; Lin, W.; Polukeev, A.; Rahaman, A.; Abdellah, M.; Chábera, P.; Pankratova, G.; Gorton, L.; Zheng, K.; Pullerits, T. Photoexcitation Dynamics in Electrochemically Charged CdSe Quantum Dots: From Hot Carrier Cooling to Auger Recombination of Negative Trions. ACS Appl. Energy Mater. 2020, 3 (12), 12525-12531.

(18) Karki, K. J.; Widom, J. R.; Seibt, J.; Moody, I.; Lonergan, M. C.; Pullerits, T.; Marcus, A. H. Coherent Two-Dimensional Photocurrent Spectroscopy in a PbS Quantum Dot Photocell. Nat. Commun. 2014, 5 (1), 1-7.

(19) Engel, G. S.; Calhoun, T. R.; Read, E. L.; Ahn, T.-K.; Mančal, T.; Cheng, Y.-C.; Blankenship, R. E.; Fleming, G. R. Evidence for Wavelike Energy Transfer through Quantum Coherence in Photosynthetic Systems. Nature 2007, 446 (7137), 782-786.

(20) Brixner, T.; Stenger, J.; Vaswani, H. M.; Cho, M.; Blankenship, R. E.; Fleming, G. R. Two-Dimensional Spectroscopy of Electronic Couplings in Photosynthesis. Nature. 2005, 434 (7033), 625-628.

(21) Seibt, J.; Hansen, T.; Pullerits, T. 3D Spectroscopy of Vibrational Coherences in Quantum Dots: Theory. J. Phys. Chem. B 2013, 117 (38), 11124-11133.

(22) Cundiff, S. T. Optical Three Dimensional Coherent Spectroscopy. Phys. Chem. Chem. Phys. 2014, 16 (18), 8193-8200.

(23) Lenngren, N.; Abdellah, M. A.; Zheng, K.; Al-Marri, M. J.; Zigmantas, D.; Žídek, K.; Pullerits, T. Hot Electron and Hole Dynamics in Thiol-Capped CdSe Quantum Dots Revealed by 2D Electronic Spectroscopy. Phys. Chem. Chem. Phys. 2016, 18 (37), 26199-26204.

(24) Karki, K. J.; Chen, J.; Sakurai, A.; Shi, Q.; Gardiner, A. T.; Kühn, O.; Cogdell, R. J.; Pullerits, T. Before Förster. Initial Excitation 
in Photosynthetic Light Harvesting. Chem. Sci. 2019, 10 (34), 79237928.

(25) Bukartè, E.; Haufe, A.; Paleček, D.; Büchel, C.; Zigmantas, D. Revealing Vibronic Coupling in Chlorophyll $\mathrm{C} 1$ by PolarizationControlled 2D Electronic Spectroscopy. Chem. Phys. 2020, 530, 110643.

(26) Mueller, S.; Lüttig, J.; Brenneis, L.; Oron, D.; Brixner, T. Observing Multiexciton Correlations in Colloidal Semiconductor Quantum Dots via Multiple-Quantum Two-Dimensional Fluorescence Spectroscopy. ACS Nano 2021, 15 (3), 4647-4657.

(27) Wang, Z.; Lei, S.; Karki, K. J.; Jakobsson, A.; Pullerits, T. Compressed Sensing for Reconstructing Coherent Multidimensional Spectra. J. Phys. Chem. A 2020, 124 (9), 1861-1866.

(28) Cassette, E.; Pensack, R. D.; Mahler, B.; Scholes, G. D. RoomTemperature Exciton Coherence and Dephasing in Two-Dimensional Nanostructures. Nat. Commun. 2015, 6, 1-7.

(29) Fuller, F. D.; Pan, J.; Gelzinis, A.; Butkus, V.; Senlik, S. S.; Wilcox, D. E.; Yocum, C. F.; Valkunas, L.; Abramavicius, D.; Ogilvie, J. P. Vibronic Coherence in Oxygenic Photosynthesis. Nat. Chem. 2014, 6 (8), 706-711.

(30) Pullerits, T.; Zigmantas, D.; Sundström, V. Beatings in Electronic 2D Spectroscopy Suggest Another Role of Vibrations in Photosynthetic Light Harvesting. Proc. Natl. Acad. Sci. U. S. A. 2013, 110 (4), 1148-1149.

(31) Butkus, V.; Zigmantas, D.; Abramavicius, D.; Valkunas, L. Distinctive Character of Electronic and Vibrational Coherences in Disordered Molecular Aggregates. Chem. Phys. Lett. 2013, 587, 9398.

(32) Paleček, D.; Edlund, P.; Westenhoff, S.; Zigmantas, D. Quantum Coherence as a Witness of Vibronically Hot Energy Transfer in Bacterial Reaction Center. Sci. Adv. 2017, 3 (9), 1-6.

(33) Collini, E.; Gattuso, H.; Bolzonello, L.; Casotto, A.; Volpato, A.; Dibenedetto, C. N.; Fanizza, E.; Striccoli, M.; Remacle, F. Quantum Phenomena in Nanomaterials: Coherent Superpositions of Fine Structure States in CdSe Nanocrystals at Room Temperature. J. Phys. Chem. C 2019, 123 (51), 31286-31293.

(34) Gellen, T. A.; Lem, J.; Turner, D. B. Probing Homogeneous Line Broadening in CdSe Nanocrystals Using Multidimensional Electronic Spectroscopy. Nano Lett. 2017, 17 (5), 2809-2815.

(35) Mukamel, S. Principles of Nonlinear Optical Spectroscopy; Oxford University Press: 1995.

(36) Hamm, P.; Zanni, M. T. Concepts and Methods of 2D Infrared Spectroscopy; Cambridge University Press: 2011.

(37) Augulis, R.; Zigmantas, D. Two-Dimensional Electronic Spectroscopy with Double Modulation Lock-in Detection: Enhancement of Sensitivity and Noise Resistance. Opt. Express. 2011, 19 (14), $13126-13133$.

(38) Konar, A.; Sechrist, R.; Song, Y.; Policht, V. R.; Laible, P. D.; Bocian, D. F.; Holten, D.; Kirmaier, C.; Ogilvie, J. P. Electronic Interactions in the Bacterial Reaction Center Revealed by Two-Color 2D Electronic Spectroscopy. J. Phys. Chem. Lett. 2018, 9 (18), 52195225 .

(39) Gelzinis, A.; Butkus, V.; Songaila, E.; Augulis, R.; Gall, A.; Büchel, C.; Robert, B.; Abramavicius, D.; Zigmantas, D.; Valkunas, L. Mapping Energy Transfer Channels in Fucoxanthin-Chlorophyll Protein Complex. Biochim. Biophys. Acta - Bioenerg. 2015, 1847 (2), 241-247.

(40) Norris, D.; Bawendi, M. Measurement and Assignment of the Size-Dependent Optical Spectrum in CdSe Quantum Dots. Phys. Rev. B - Condens. Matter Mater. Phys. 1996, 53 (24), 16338-16346.

(41) Caram, J. R.; Zheng, H.; Dahlberg, P. D.; Rolczynski, B. S.; Griffin, G. B.; Dolzhnikov, D. S.; Talapin, D. V.; Engel, G. S. Exploring Size and State Dynamics in CdSe Quantum Dots Using Two-Dimensional Electronic Spectroscopy. J. Chem. Phys. 2014, 140 (8), 084701.

(42) Prezhdo, O. V. Multiple Excitons and the Electron-Phonon Bottleneck in Semiconductor Quantum Dots: An Ab Initio Perspective. Chem. Phys. Lett. 2008, 460, 1-9.

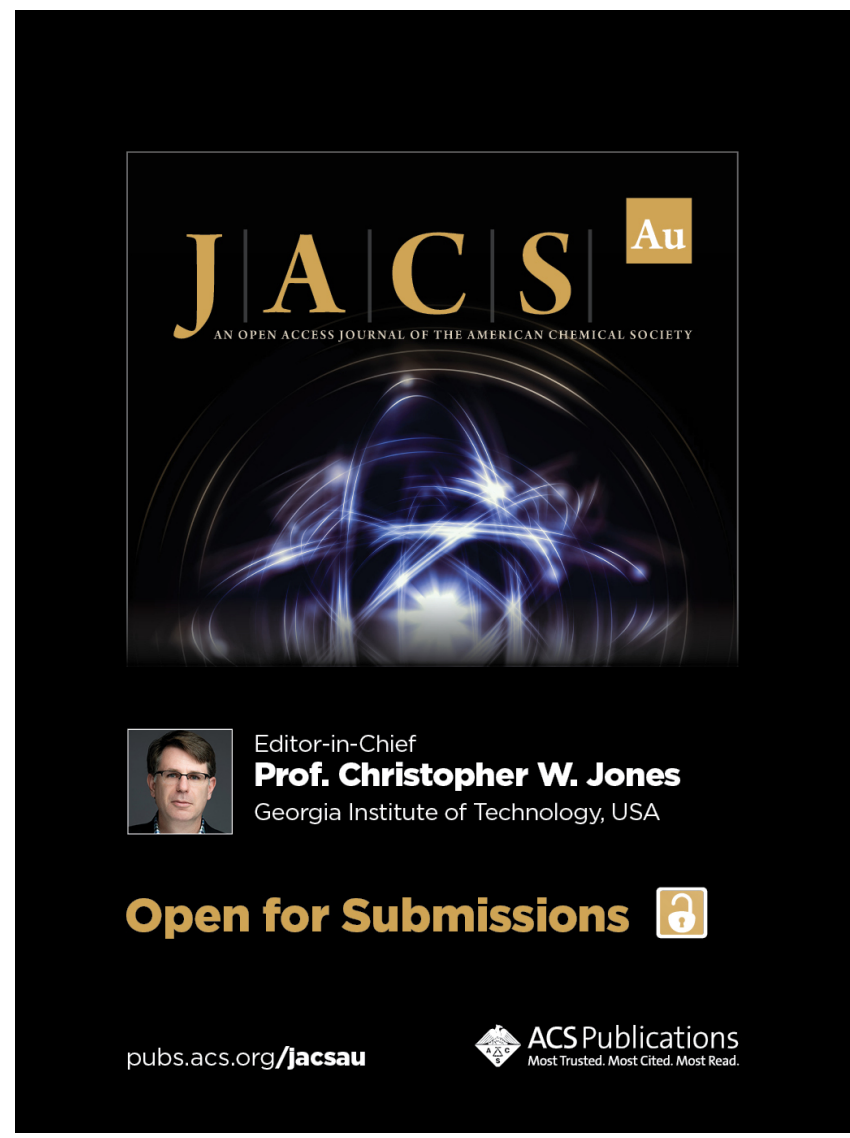

